\title{
Multi-Ensayos
}

Vol. 8, núm. 15

ISSN: 2412-3285

https://multiensayos.unan.edu.ni

DOI: https://doi.org/10.5377/multiensayos.v8i15.13190

\section{Construcción de la identidad en el sujeto contemporáneo}

\section{Construction of identity in the contemporary subject}

Ángel Alejandro Gutiérrez Portillo'

Recibido: 16 de julio de 2021. Aceptado: 12 de noviembre de 2021

\section{RESUMEN}

A partir de la década de 1970 múltiples intelectuales se percataron y comenzaron a discutir acerca de la poca incidencia que tenían las instituciones sociales en los sujetos contemporáneos. Desde tal punto de vista, se viene observando que la metamorfosis sociocultural actual se expresa a través de un lenguaje plural, diverso, variado, mediante el cual se revaloriza y se fomenta el cuidado del medioambiente. Asimismo, porque se realza la individualidad de las personas, quienes concentran sus relaciones sociales a partir de las tecnologías de información y comunicación, promoviendo la globalización de una cultura consumista. Esta mutación sociocultural nos ha dirigido irremediablemente hacia un tipo de sociedad peculiarmente nueva, lo que ha generado una compleja transformación sociocultural en el sujeto contemporáneo, donde el tema de la identidad social vuelve a emerger como un fenómeno social novedoso en los estudios sociológicos.

Palabras claves: metamorfosis sociocultural; construcción; identidad; sujeto.

\section{ABSTRACT}

Starting in the 1970s, multiple intellectuals noticed and began to argue about the little impact that social institutions had on contemporary subjects. From this point of view, it has been observed that the current sociocultural metamorphosis is expressed through a plural, diverse, varied language, through which the care of the environment is revalued and promoted. Likewise, because the individuality of people is enhanced, who concentrate their social relationships based on information and communication technologies, promoting the globalization of a consumer culture. This sociocultural mutation has inevitably directed us towards a peculiarly new type of society, which has generated a complex sociocultural transformation in the contemporary subject, where the issue of identity re-emerges as a novel social phenomenon in sociological studies.

Keywords: sociocultural metamorphosis; construction; identity; subject.

1 Profesor Investigador, Titular B Tiempo Completo, de la Universidad Juárez Autónoma de Tabasco, Posdoctor en ciencias antropológicas por la Universidad Autónoma Metropolitana, Posdoctor en estudios sociales por El Colegio de la Frontera Norte. Doctor en antropología por la Universidad Nacional Autónoma de México. Estudioso de las identidades sociales, del fenómeno médico y religioso. Perfil deseable del Programa para el Desarrollo Profesional Docente. Miembro del Sistema Nacional de Investigadores del CONACyT. ORCID: http://orcid.org/0000-0002-7349-1221; gupalex@live.com.mx

(C) 2022 Revista Multi-Ensayos. 


\section{INTRODUCCIÓN}

Desde hace varias décadas, diversos autores vienen hablando sobre la decadencia de las instituciones sociales. Esta perspectiva sostiene que las transformaciones sociales contemporáneas se caracterizan por tener un lenguaje heterogéneo que no es univoco ni estable (Derrida, 1986), por revalorizar y promover el cuidado del medio ambiente (Sauvé, 1999), por engrandecer la individualidad de los sujetos (Bajoit, 2003), por estar centrada en las tecnologías de información y comunicación (Castells, 2001, 2003 y 2005; Lyotard, 2006), por suscitar la globalización de una cultura consumista (Bauman, 1996, 2001 y 2007).

Estos cambios sociales nos han conducido irreversiblemente hacia un tipo de sociedad cualitativamente nueva, ocasionado una profunda mutación cultural en el sujeto contemporáneo, donde el tema de la identidad ${ }^{1}$ constituye un hecho novedoso y sintomático en los estudios sociológicos (Bajoit, 2003).

Para dar cuenta de este paradigma sociológico, primero hablaremos sobre el concepto de institución, con la finalidad de explicar y discutir lo que son las instituciones sociales, las cuales han decaído por la poca incidencia que tienen en la conformación de la identidad de los sujetos contemporáneos, quienes, hoy en día, construyen su identidad en la dimensión subjetiva que ellos mismos han elaborado, respecto al punto donde se ubiquen entre lo establecido socialmente (el determinismo) y la autonomía (la libertad). ${ }^{2}$

\section{El concepto de institución}

La noción de institución, entendida de forma habitual, designa los mecanismos de orden social y cooperación que procuran regular el comportamiento de un grupo de individuos (puede ser de una colectividad únicamente o coincidir con una sociedad entera). Las instituciones establecen todas las actividades regidas por anticipaciones estables y recíprocas (se concibe como institución a las organizaciones, las costumbres, los hábitos, las reglas del cine, las religiones, etcétera). Por ejemplo, la familia es una institución como la Iglesia, el Estado, la Universidad, la Federación Mexicana de Fútbol, la Organización Mundial de la Salud, las recetas de cocina tradicional, entre otras más, es decir, las instituciones son maneras de ser, de pensar, son objetos, hechos sociales ${ }^{3}$, los cuales se remiten a un conjunto de instituciones. Para ampliar nuestra noción de institución, consideremos la siguiente definición: "Se puede llamar institución, sin desnaturalizar el sentido de esta palabra, a todas las creencias y a todos los modos de conducta instituidos por la colectividad" (Durkheim, 1997, p. 23).

En el sentido sociológico de Durkheim, una institución no es una persona ni un grupo, es parte de la cultura, un sector estandarizado del modo de vida de una sociedad.

1 En este trabajo comprendemos por identidad al conjunto de rasgos culturales de un individuo que lo distingue de otras personas del mismo entorno como de otros contextos sociales.

2 En la sociedad contemporánea los sujetos construyen su identidad a partir de lo establecido por la sociedad, pero son libres para pensar, decidir y actuar, en cuanto a la autonomía que los caracteriza como individuos sociales.

3 Entendemos por hecho social a "toda manera de hacer, fija o no, susceptible de ejercer sobre el individuo una coacción exterior; o también, que es en general dentro de la extensión de una sociedad dada a la vez que tiene una existencia propia, independiente de sus manifestaciones individuales" (Durkheim, 1997, p. 36). 
Por su parte, desde la perspectiva de Radcliffe-Brown (1980) la institución se concibe como "una norma de conducta establecida y reconocida como tal por un grupo social en cuyo seno es, por ello mismo, una institución" (p. 113).

Ambas definiciones comparten elementos de significado, pero como bien lo alude Douglas (1996) las instituciones no son sólo hechos y prácticas colectivas, sino también marcos cognitivos y morales dentro de los cuales se desarrollan los pensamientos individuales.

Esto quiere decir que la lengua sería una de las primeras instituciones, porque está asociada a los modos de pensamiento y conocimiento que persisten en tanto son compatibles con las experiencias individuales y colectivas. En ese sentido, podemos señalar que son instituciones la poesía, la narrativa y la dramaturgia.

Por su parte, si nos remitimos al Diccionario de la Real Academia Española (2011), la palabra institución tiene once significados:

1. Establecimiento o fundación de algo.

2. Cosa establecida o fundada.

3. Organismo que desempeña una función de interés público, especialmente benéfico o docente.

4. Cada una de las organizaciones fundamentales de un Estado, nación o sociedad. Institución monárquica, del feudalismo.

5. De sus. Instrucción, educación, enseñanza.

6. Colección metódica de los principios o elementos de una ciencia, de un arte, etcétera.

7. Órganos constitucionales del poder soberano en la nación.

8. Acción de conferir canónicamente un beneficio. canónica.

9. Acción de poner a alguien en posesión de un beneficio. corporal.

10. Nombramiento que en el testamento se hace de la persona que ha de heredar. $\sim$ de heredero.

11. Tener en una ciudad, empresa, tertulia o cualquier otra agrupación humana el prestigio debido a la antigüedad o a poseer todos los caracteres representativos de aquella. ser alguien una.

Con estas de acepciones podemos decir que una institución es un establecimiento u organismo que realiza una labor social de tipo educativo y cultural, como los institutos de enseñanza, investigación o los propios museos. Del mismo origen vienen instrucción, instructor e institutriz.

Sin embargo, cabe precisar que el término Institución se aplica por lo general a las normas de conducta consideradas importantes para una sociedad, como las particulares organizaciones formales de gobierno y servicio público. El concepto institución no tiene que ser un lugar físico. Una institución puede ser conducir un automóvil por la derecha en ciertos países. También puede ampliarse el concepto para designar a una persona de especial consideración, veneración y respeto, por ejemplo, Pedro Armendáriz es una institución en el cine mexicano o Gustavo Cerati es una institución en el rock latinoamericano. Hay muchos grados de instituciones, están desde las formales hasta las informales. Las primeras son aquellas que están reconocidas socialmente por una autoridad, mientras que las segundas son aquellas que se establecen al interior de un grupo y son ajenas al resto de la sociedad. 
De acuerdo con el sociólogo norteamericano Joseph Fichter (1994) el concepto de Institución tiene las siguientes características:

a. Las instituciones, aun sin necesidad de personificarlas, son intencionales, en cuanto que cada una de ellas tiene como objetivo o fin la satisfacción de una necesidad social. Son los principales modos de comportamiento mediante los cuales se asocian personas entre sí para hacer determinadas cosas.

b. Tienen un contenido relativamente permanente. Las pautas, roles y relaciones que realizan las personas en una determinada cultura son tradicionales y duraderos. Como toda obra humana, están sujetos a modificaciones, pero las modificaciones institucionales son relativamente lentas.

c. La institución está estructurada, u organizada, o coordinada. Los componentes tienden a mantenerse en cohesión y a reforzarse mutuamente. Esto se sigue del hecho de que los roles y las relaciones sociales son en sí mismos combinaciones estructuradas de pautas de comportamiento.

d. Cada institución es una estructura unificada en el sentido de que funciona como una unidad, aunque sea interdependiente con otras instituciones. Ninguna institución puede separarse completamente de las otras instituciones o roles, pero cada uno de ellos funciona como una serie de identificable de pautas de conducta.

e. La institución está necesariamente cargada de valor, por cuanto sus uniformidades de conducta repetidas se volvieron códigos normativos de conducta, algunos de ellos expresados en reglas y leyes escritas, pero limitados en su mayoría a ejercer una presión social subconsciente sobre la gente.

Para nuestro propósito es suficiente con entender como institución a "un agrupamiento configurado por reglamentos establecidos racionalmente" (Weber, 1987, p. 55). Es decir, una institución se conforma por diversos elementos que constituyen las pautas de comportamiento compartidas por una colectividad, las cuales pretenden satisfacer alguna necesidad básica de un grupo sui generis.

Esta definición tan amplia y puntual incluye todos los significados que venimos esbozando. Por ello, es la que utilizaremos a lo largo del presente trabajo.

\section{Las instituciones sociales}

En este inciso hablaremos de algunas peculiaridades que tienen las instituciones sociales como la familia, la iglesia, la escuela y el Estado. ${ }^{4}$ Esto nos permitirá dilucidar la naturaleza y el propósito que de ellas emana.

En primer lugar, el origen de las instituciones sociales nos remite al seno familiar, como una necesidad de socialización entre las personas. Por socialización se concibe a "todas y cada una de las instancias a través de las cuales un sujeto humano se integra e incorpora las consignas y determinaciones de la estructura social en la que interactúa. Más precisamente: la socialización incluye todas las instancias a través de las cuales un sujeto humano se hace individuo. Ser individuo implica "individualizar" en una persona aquellas características generales que connotan una estructura social" (Kaminsky, 2001, p. 11).

4 El orden en el que se presentan las particularidades no radica en la importancia de cada una de ellas, sino más bien en las necesidades del propio escrito. 
Esta tesis postula que las personas sólo pueden desarrollarse y poseer identidades plenamente acabadas conforme cada cual capta y refleja, en su experiencia individual, aquellas actitudes y/o actividades sociales organizadas que representan las instituciones sociales. No obstante, la idea de institución social no significa únicamente que la socialización consista en inscribir una cultura en un individuo; asimismo designa una manera única de llevar a cabo ese trabajo sobre los otros. Por ello, las instituciones sociales implican la transformación de valores y principios morales en los sujetos:

En primer lugar, existe un carácter común a todos los valores y principios que comúnmente se denominan morales: que todos existen conforme a reglas preestablecidas. Conducirse moralmente significa actuar siguiendo una norma, determinando la conducta a observar en el caso dado, incluso antes de vernos obligados a tomar partido. El dominio de la moral es el dominio del deber, y el deber es una acción prescrita (...) podemos, pues, decir que la moral es un sistema de reglas de acción que predeterminan la conducta (Durkheim, 2001, p. 30-31).

Es decir:

[La moral] es un conjunto de reglas definidas; es como un conjunto de moldes, de contornos definidos, en los cuales debemos verter nuestra acción. No tenemos que construir estas reglas en el momento en que hay que actuar, deduciéndolas de principios más elevados. Existen, están ya terminadas, viven y funcionan alrededor nuestro. Son la realidad en su forma concreta (Durkheim, 2001, p. 34).

Desde tal perspectiva, la moral es algo constante, siempre y cuando la observación no se extienda en períodos prolongados, porque la moral por sí misma presupone cierta cualidad para repetir los mismos actos, porque fomenta y recrea hábitos.

Por tal motivo, para el fundador de la sociología francesa Émile Durkheim (2001) la moral "consiste en un conjunto de reglas definidas y especiales que determinan imperativamente la conducta" (p. 41). Este sentido de puntualidad y de autoridad, corresponde a dos aspectos de un mismo estado de ánimo. En primer lugar, el espíritu de disciplina, porque la moral es una regla ${ }^{5}$ que determina las acciones de los sujetos, imponiéndole al individuo cierta disposición para vivir condicionado. "Al ser regular el deber, se repita siempre uniforme. Incluso monótonamente. Los deberes no son acciones brillantes realizadas de tiempo en tiempo, en momentos de crisis intermitentes. Los verdaderos deberes son cotidianos, y el curso natural de la vida los vuelve atraer periódicamente. Aquellos para quienes el gusto por el cambio y la diversidad llegan hasta horrorizarlos ante cualquier uniformidad, se arriesgan a ser moralmente incompletos. La regularidad es lo análogo moral de la periodicidad orgánica" (p. 42). En segundo lugar, las reglas morales no son simplemente otra denominación de los hábitos interiores, pues determinan la conducta desde afuera e imperativamente, porque los actos no son ajustados a una conducta individual sino colectivamente, ya que está predeterminada y socialmente aceptada. En otras palabras, "es preciso que el individuo esté constituido de manera tal que sienta la superioridad de las fuerzas morales, cuyo valor es más elevado que el suyo, y se incline ante ellas" (p. 42).

5 La moral es una regla, pero una regla siempre es flexible, no puede ser fija e invariable ni que la coloque por encima de los intereses individuales. 
Si la moral moldea nuestra conducta desde la colectividad, entonces uno como individuo está prescrito a pertenecer a distintas instituciones sociales. ${ }^{6}$ Es por ello, que todo individuo desde antes de nacer y en el transcurso de su vida (hasta en la muerte) ${ }^{7}$ pertenece a una institución social, la familia, la iglesia, la escuela, el Estado, etcétera, pero cada una de estas instituciones tiene formas únicas de regular la socialización de las personas.

Sólo hay fines verdaderamente morales en los fines colectivos; no hay móvil verdaderamente moral fuera de la adhesión al grupo. Pero al estar ligado a la sociedad de la cual se es parte, resulta psicológicamente imposible no hallarse ligado a la vez a los individuos que la componen y en quienes la sociedad se realiza. Pues, aunque la sociedad es algo distinto al individuo, pues no está íntegramente en nosotros, no existe sin embargo ninguno de nosotros en quien no se encuentre un reflejo de ella (Durkheim, 2001, p. 95).

Ejemplo de esto, lo encontramos en la familia (en toda la extensión de la palabra) como institución primaria de la sociedad en México, puesto que se encarga de establecer valores y principios morales a través de un sistema de normas que regulan las interacciones sociales entre los individuos que la integran.

Un aspecto importante que debemos considerar es que durante mucho tiempo la iglesia impregnó de sus valores y principios a la familia en México. Hasta podemos decir que la iglesia fue la forma embrionaria de la escuela, puesto que los sacerdotes fungían como docentes. ${ }^{8}$ De hecho, la escuela en México no es sólo un lugar de aprendizaje ni un espacio donde el profesor enseña, también es un espacio moral, porque la escuela es un ambiente moral y el docente es un ser moral impregnado de ciertas ideas, de ciertos sentimientos, que envuelve tanto a los estudiantes como al maestro (Dubet, 2006).

Por esta razón es que los pueblos han visto, durante siglos, en las reglas de la moral, órdenes emanadas de la divinidad. Y es porque una regla no es una simple manera habitual, sino una manera tal de actuar, que no nos sentimos libres como para modificarla a nuestro gusto. Está, en alguna medida, y en la medida en que es una regla, sustraída a nuestra voluntad. Hay en ella algo que nos resiste, que nos sobrepasa, que se nos impone, que nos obliga (Durkheim, 2001, p. 36).

Para fundar su doctrina en México, la Iglesia católica resaltó un doble compromiso social. Por una parte, estuvo a favor de defender el universalismo (el de Roma), contra la diversidad de dogmas religiosos. Por otra, apeló a un sujeto, a una disposición moral propia de todos los individuos, que pertenecía sólo a los individuos y a Dios; sobre ese sujeto la Iglesia católica estableció su reino (Dubet, 2006).

6 Cabe señalar que la moral moldea la conducta de los sujetos. Pero no todos los sujetos son moldeados de la misma forma por la moral para construir su identidad, puesto que está adquiere diversos valores por la carga sociocultural de cada individuo.

7 Este tipo de adscripción se da regularmente en aquellos individuos que fueron feligreses de alguna doctrina religiosa y que se les recuerda como parte de esa institución. Por ejemplo, en la Iglesia católica se le hace una misa para recordar al "santo" difunto.

8 "No existe ningún pueblo en el que no exista cierto número de ideas, de sentimientos y de prácticas que la educación tiene que inculcar a todos los niños indistintamente, sea cual fuere la categoría social a la que pertenecen. Hasta en esos países en los que la sociedad está dividida en castas cerradas la una a la otra, existe siempre una religión común para todos y, por consiguiente, los principios de la cultura religiosa, que pasa a ser entonces fundamental, son los mismos para toda la masa de la población. Aun cuando cada casta y cada familia tenga sus dioses particulares, existen también divinidades generales, reconocidas por todos y a las que todos los niños aprenden a venerar" (Durkheim, 1976, p. 96). 
La iglesia "inventa" la escuela porque cuenta con un proyecto de dominación universal sobre las almas. Para ello hace falta una Escuela: para inculcar prácticas, un adiestramiento maquinal es suficiente o eficaz; pero las ideas, los sentimientos, no pueden comunicarse si no es con la voz de la razón (Durkheim, 2001, p. 30).

Esa conquista de las almas no podría ser únicamente un adiestramiento; también era una revelación del sujeto, porque él interiorizaba una polaridad de pensamientos, surgidos de la iglesia y del Estado. La escuela fue entonces mediatriz de muchos términos: el mundo cristiano y el mundo pagano, el mundo soberano y el mundo de los hombres, la cultura universal, luego nacional, y las culturas específicas (Dubet, 2006).

Durante varios siglos en México, la iglesia engendró en la escuela una forma de racionalización del individuo, que trajo consigo el proyecto de laicización del Estado moderno. ${ }^{9}$ Al establecerse el Estado como el procurador del bienestar social, se encargó de instituir los nuevos valores y principios en la familia como en la escuela. $Y$ aunque el Estado ha tratado de escindir las concepciones terrenales de las sagradas, estas han persistido, ya que mantener la distancia y la influencia de la Iglesia sobre el Estado laico siempre ha sido difícil y complicado.

[En el caso de México] el proceso de separación Iglesia-Estado, establecido con las reformas juaristas de la segunda mitad del siglo pasado, inició un amplio período de distanciamiento y en ocasiones un profundo conflicto entre las instituciones religiosas y las del Estado mexicano, como sucedió durante el movimiento cristero (Salles y Valenzuela, 1997, p. 73).

Esto no quiere decir que ambas instituciones hayan dejado de seguir permeando con sus valores y principios morales a la sociedad en general. En cierta medida eso sería imposible, porque "el conocimiento de la religión es indispensable en la educación de Estado, siendo que la historia de las religiones nos ayuda a comprender nuestra historia y el presente. Lo que sí sería importante es que la escuela que pretende ser laica debe ser al mismo tiempo tolerante en materia de opiniones y opciones religiosas, y no como aquella en la que la enseñanza religiosa está basada en una religión de Estado. La instrucción educativa no debe ignorar la diversidad de creencias y prácticas religiosas particulares, pero tan poco debe olvidar que si su enseñanza está mutilada crea desigualdades sociales" (Touraine, 2005, p. 221).

Una vez reconocidas las cualidades de la familia, la iglesia, la escuela y el Estado, podemos aseverar entonces que, las instituciones fundan sobre valores, principios, dogmas, mitos, creencias laicas o religiosas, la tradición de un hilo de memoria que funge como guía entre las sociedades pasadas y las sociedades contemporáneas, con la finalidad de coadyuvar en la construcción de la identidad en los sujetos. No obstante, cada institución tiene una forma particular de moldear a los individuos. Por ello, las instituciones invocan principios y valores que no se presentan como simples reflejos de una sociedad, estos se construyen sobre generalidades. Como bien lo menciona Dubet (2006) "las instituciones no pueden cumplir su tarea sino en la medida en que se fundan los valores percibidos como universales" (p. 50).

9 Con la Independencia de México en 1810, inició el proceso de laicización. Sin embargo, fue con las leyes de reforma de 1859, cuando el poder -que durante varios siglos tuvo la iglesia- pasó a formar parte del Estado mexicano (Gutiérrez, 2017). 
Para Durkheim (1990) "los individuos que componen el mundo moderno son de tal modo diferentes unos a otros que casi no tienen nada en común entre ellos, salvo la calidad de hombre original. No pueden conservar la homogeneidad indispensable para todo consenso social más que con la condición de ser también parecidos por el único aspecto en que se parecen todos, es decir, en tanto que son seres humanos" (p. 100).

Se trata de una cultura universal, que a priori vale para todos los seres humanos, que ancla la subjetividad de los individuos porque les permite ser más críticos, más reflexivos y conscientes de sí mismos. Sobre este paradigma, dice Durkheim, el contenido de la cultura difundida cuenta menos que la forma de su difusión, que disciplina propiamente dicha. Él cree más en la eficacia de la forma escolar que en los programas escolares. Al respecto menciona lo siguiente:

En la escuela elemental el niño queda encerrado en una moral objetiva. Está como hipnotizado por el maestro, aprende a controlar su cuerpo y sus pasiones infantiles, adquiere hábitos que serán más que hábitos. El trabajo escolar se centra en la repetición de ejercicios presentados como otros tantos ritos formales: aprender de memoria, recitar, copiar, ponerse en fila, responder cuando se hacen preguntas (...) en la enseñanza secundaria, esta disciplina se diversifica, los múltiples profesores reemplazan al maestro único. Los ejercicios son menos escolares; a medida que se incrementan los estudios la disciplina se distiende porque los alumnos ya están disciplinados (Durkheim, 2001, p. 145).

Aunque los principios y valores institucionales son universales, pueden variar sensiblemente entre una cultura y otra. Por ejemplo, los docentes de las primarias de la Ciudad de México se centran más en la trasmisión de los saberes que sus colegas de Chiapas o Tabasco, quienes se preocupan más por la integridad del alumno. No obstante, en todos los casos la matriz institucional debería enseñar al menos que no debe hacerlo bajo un marco disciplinar, porque su objetivo es socializar al individuo y construirlo en sujeto. Sin embargo, esto es una paradoja, porque la creencia en la continuidad de dicho proceso reside en la esencia de la institución. Por una parte, la institución socializa al individuo tal cual es, le inculca un habitus ${ }^{10}$ y una identidad conforme a los requisitos de la vida social. Por otra parte, como apela a principios y valores universales, las instituciones arrancan al individuo de la integración social, configurando un sujeto capaz de dominar y construir su libertad por la gracia de la fe o de la razón (Dubet, 2006).

La educación ha variado infinitamente, según los tiempos y según los países, En las ciudades griegas y latinas la educación intentaba adiestrar al individuo para que se subordinase ciegamente a la colectividad, para que se convirtiera en una "cosa" de la sociedad. Hoy, la educación se esfuerza en hacer de ella una persona autónoma (Durkheim, 1976, p. 91-92).

10 Para Bourdieu (2007) "Ios condicionamientos asociados a una clase particular de condiciones de existencia producen habitus, sistemas de disposiciones duraderas y transferibles, estructuras estructuradas predispuestas a funcionar como estructuras estructurantes, es decir, como principios generadores y organizadores de prácticas y de representaciones que pueden ser objetivamente adaptadas a su meta sin suponer el propósito consciente de ciertos fines ni el dominio expreso de las operaciones necesarias para alcanzarlos, objetivamente "reguladas" y "regulares" sin ser para nada el producto de la obediencia a determinadas reglas, $y$, por todo ello, colectivamente orquestadas sin ser el producto de la acción organizadora de un director de orquesta" (p. 86). 
Este es el escenario donde la socialización y la subjetivación se concatenan en un mismo proceso que tiene diferentes direcciones y objetivos. Ese hecho por sí mismo, es lo que ha ocasionado que hoy en día se agudice la decadencia de las instituciones sociales, por una parte, y las crisis identitarias, por la otra.

\section{La decadencia de las instituciones sociales}

Desde hace cuatro décadas las instituciones sociales han colapsado, porque la gran fuerza de ellas radicaba en creer y hacer creer en la homogeneidad de los valores y de los principios universales, que instituían en las sociedades tradicionales para generar un sentido de pertenencia y unidad, donde se configuraba la identidad de cada persona. ${ }^{11}$

El sentido (Sinn) se constituye en la conciencia humana: en la conciencia del individuo, que está individualizado en un cuerpo vivo (Leib) y ha sido socializado como persona (...) las operaciones generales de la conciencia a partir de las cuales se construye las múltiples significaciones de la experiencia y de la acción en la existencia humana se pueden observar en la constitución histórico social de la identidad personal (Berger y Luckmann, 1997, p. 30-31).

Este desmoronamiento de las instituciones sociales ha ocasionado que el mundo en el que estamos inmersos se encuentre en una profunda mutación cultural del individuo moderno (Bell, 1991; Giménez, 1996 y 1997; Berger y Luckmann, 1997; Harvey, 1998; Dubet y Martuccelli, 2000; Dubar, 2002; Bauman, 1996, 2001 y 2007; Bajoit, 2003; Martín-Barbero, 2004; Inglehart, 2005; Touraine, 2004 y 2005).

La situación actual emergió de la disolución radical de aquellas amarras acusadas -justa o injustamente- de limitar la libertad individual de elegir y de actuar. La rigidez del orden es el artefacto y el sedimento de la libertad de los agentes humanos. Esa rigidez es el producto general de "perder los frenos": de la desregulación, la liberalización, la "flexibilización", la creciente fluidez, la liberación de los mercados financiero, laboral e inmobiliario, la disminución de las cargas impositivas, etc. (Bauman, 2004, p. 4).

Tal cambio en la modernidad ha implicado que las personas se relajen de los determinismos institucionales, es decir, que los condicionamientos sociales en todos los campos de la vida social ya no son tan directos, rígidos, represivos, asertivos y exigentes como lo eran antes (las maneras instituidas de pensar, de decir y de hacer, interiorizadas por la socialización) se establecen actualmente menos en las conductas de la gente. ${ }^{12}$

Ser sujeto es ser capaz de construir, a lo largo de la vida, relaciones de conocimiento mutuo. Ser sujeto es primordialmente rechazar, tanto para uno mismo como para los demás, las relaciones de dominación, de servidumbre, de autoridad impuesta arbitrariamente, de desprecio y de subordinación personal (Dubar, 2002, p. 252).

11 "Uno de los efectos más claros de la crisis de la modernidad, del agotamiento de los proyectos históricos y del final de las ideologías se ha manifestado en la irrupción inusitada de la cuestión de la identidad. La interrogante acerca de quiénes somos se hace con mayor insistencia y su respuesta en lo social y lo colectivo resultan insatisfactorias y nos tenemos que hacer la pregunta en terrenos cada vez más personales e individuales. Dicha dimensión personal, por sí sola, resulta insuficiente para dar cuenta de la pregunta básica acerca del ser, por lo que todo intento serio que quiera enfrentarse al problema tiene que trabajar en la relación entre la identidad personal y la colectiva" (Tejeda, 2005, p. 172).

12 simbólicas" (Dubar, 2002, p. 236-237). 
Por ello, los individuos modernos apelan cada día más a su autonomía, a elegir, a decidir por sí mismos, a contar con su imaginación, con su iniciativa y con su creatividad. Esta libertad mayúscula, hace que las personas sean "más actores en sus relaciones con los otros, más sujetos en sus relaciones con ellos mismos $\mathrm{y}$, por lo mismo, conducirse más como individuos singulares, y no ya como individuos uniformizados por sus posiciones sociales" (Bajoit, 2008, p. 15).

Este individuo se vuelve más expuesto y más fuertemente sometido a una serie de pruebas concernientes a su obligación de ser libre y soberano (Dubet y Martuccelli, 2000, p. 19).

Como lo ha mencionado en múltiples ocasiones Bajoit (2003, 2008 y 2009) hoy como en ninguna otra época, las personas se han convertido en verdaderos Individuos, Sujetos, Actores "el Gran ISA". ${ }^{13}$ Esta mutación cultural del individuo nos ha conducido "de la tiranía de la Razón a la tiranía del Gran ISA ${ }^{14}$, que nos impone una nueva "Tabla de la Ley" donde figuran una serie de derechos-deberes como el de autorrealización personal, el de libre elección, el de la búsqueda del placer inmediato y el de seguridad frente a los riesgos y amenazas exteriores. Pero como estos derechos-deberes son en parte contradictorios y están sembrados de trampas, su cumplimiento genera en los individuos tensiones psíquicas que afectan el equilibrio entre las diversas zonas de su identidad personal" (Bajoit, 2009, p. 9). Esto es a lo que llama Giménez (1996) y Dubar (2002) "la crisis de las identidades". ${ }^{15}$

La crisis de los individuos sobrecargados de problemas para cuya solución no encuentran ya ninguna ayuda en las instituciones, ni civiles, ni jurídicas, ni religiosas (Touraine, 2005, p. 15).

Para el sociólogo Gilberto Giménez "esta crisis afecta, por un lado, a todo el sistema de identidades tradicionales en los países en desarrollo bajo el desafío de la modernización; y por otro, al sistema de identidades ideológicas, políticas y hasta religiosas que se habían configurado en el escenario internacional a partir de la segunda guerra mundial y que han terminado por desmoronarse bajo los embates de la guerra fría" (1996, p. 183). ${ }^{16}$

Por su parte, Dubar (2002) menciona que las crisis de las identidades se engendraron por el desplazamiento y la ruptura de las formas identitarias (comunitarias) por las societarias (individuales). Para ser más explícito comenzaré explicando en qué consisten dichas representaciones. Las formas identitarias suponen "la

13 Según mi interpretación de "lo que hoy está ocurriendo", esta mutación se traduce en la mente de un número creciente de individuos por la credibilidad en constante ascenso de un nuevo "Dios reinante" al que yo llamo -con un toque de ironía para guardar mis distancias- "el Gran ISA": ¡el gran Individuo, Sujeto, Actor! (Bajoit, 2009, p. 10). 14 Este nuevo Dios se parece demasiado a sus antepasados, al grado que, es construido por las personas a través de su creer y en la práctica de sus relaciones sociales. Por lo tanto, es considerado como un personaje trascendente destinado a dar sentido (orientación y significado) a la existencia y a las conductas de los individuos en todos los campos relacionales de su vida común.

15 "Los estudios de las identidades sociales constituyen un tópico de interés para las ciencias políticas, la sociología y la antropología. En primer lugar, porque los políticos están preocupados por las particularidades nacionales y locales en un mundo que tiende a la globalización y a la homologación cultural. En segundo lugar, por la natural sensibilidad de las disciplinas sociales a la manifestación de una nueva crisis social que podríamos llamar crisis general de identidades" (Giménez, 1996, p. 183).

16 "El proceso de globalización ha generado, por un lado, nuevas identidades como resultado de la apertura de fronteras y por otro, la reivindicación de lo propio, por parte de ciertos grupos que se resisten a abandonar su cultura. Los Estados-nación enfrentan un gran desafío: la búsqueda de mecanismos a través de los cuales puedan convivir con esquemas simbólico-culturales diferentes y hasta contradictorios" (Mercado y Hernández, 2010, p. 230). 
creencia en la existencia de agrupaciones denominadas comunidades consideradas como sistemas de lugares y nombres preasignados a los individuos y que se reproducen idénticamente a lo largo de las generaciones" (p. 13). Es decir, en la forma identitaria cada individuo pertenece a su comunidad porque ahí nació, tiene una adscripción por natalidad, es lo que se conoce como identidad residencial (Bartolomé, 2004). En cambio, las formas societarias, suponen la existencia de "colectivos múltiples, variables y efímeros a los que los individuos se adhieren por periodos limitados y que proporcionan recursos de identificación que se plantean de manera diversa y provisional" (Dubar, 2002, p. 13). En otras palabras, en la forma societaria, cada persona posee múltiples pertenencias que pueden cambiar con el curso de la vida.

En resumen, las formas comunitarias son aquellas que determinan a cada persona sus normas, reglas, papeles y estatus reproducidos de generación en generación. Mientras que las formas societarias son aquellas que no determinan nada, pero ofrecen a los individuos oportunidades, recursos, señas y un lenguaje para la construcción de este, permitiendo así la diferenciación y singularización de cada persona. ${ }^{17}$

Ahora, volviendo al punto, si las formas identitarias y las societarias se pusieran en un mismo plano se hibridarían. ${ }^{18}$ Sin embargo, el problema reside en que cada día son más grandes las separaciones entre los universos culturales de las diferentes generaciones, ocasionando verdaderas fracturas culturales que alcanzan en profundidad a las identidades individuales y sociales. Es lo que hoy vemos como un desfase generacional de concebir el mundo. Lo que para los padres puede estar bien, para los hijos no y viceversa. Podemos hablar entonces de una crisis de las identidades en el sentido de desestabilización de la disposición anterior de las formas identitarias.

Esta mutación de modelo cultural al que están enfrentándose los sujetos modernos implica intensa y fundamentalmente en "ser uno mismo" (Dubet y Martuccelli, 2000). Y ser uno mismo es inscribir la historia individual y social para tener una identidad personal, aunque ésta se fugaz (la identidad personal y social no es estática, se transforma de acuerdo con a sus contenidos históricos). ${ }^{19}$

En otras palabras, "ser tú mismo" en el mundo contemporáneo implica (de) construirte para (re)construirte una y otra vez, lo que conlleva siempre a una crisis, porque todo cambio, por mínimo que sea o parezca, trae consigo en el individuo un desajuste o, mejor dicho, una crisis (Touraine, 2005), en este caso, identitaria (Giménez, 1996; Dubar, 2002).

Cuando ciertos hábitos seculares se desmoronan, cuando ciertos tipos de vida desaparecen, cuando ciertas viejas solidaridades se deshacen, entonces ciertamente suele producirse una crisis de identidad (Lévi-Strauss, citado en Giménez, 1996, p. 184).

17 "Para que una identidad se atribuya tiene que ser reconocida por los demás en contextos de interacción y de comunicación, lo que requiere una "intersubjetividad lingüística" que moviliza tanto la primera persona (el hablante) como la segunda (el interpelado, el interlocutor)" (Habermas, 1992b, p. 144).

18 Por hibridación comprendemos a "Ios procesos socioculturales en los que estructuras o prácticas discretas, que existían en forma separada, se combinan para generar nuevas estructuras, objetos y prácticas" (García, 1990, p. 3).

19 La identidad personal es la trayectoria social, que es también una historia subjetiva. Cada individuo posee varias identidades en el sentido de posiciones (padre, profesor, hermano, amigo, etc.) en las categorías oficiales (Dubar, 2002). 
Como bien lo alude Berger, y Luckmann:

Éste es el lado subjetivo de la precariedad de todos los mundos construidos por el hombre. La dificultad de mantener en marcha un mundo se expresa psicológicamente a través de la dificultad de que dicho mundo se mantenga plausible. El mundo se construye en la conciencia del individuo por el dialogo con aquellas personas más significativas de entre sus semejantes (tal como padres, maestros, camaradas). Si esta conversación se interrumpe (la esposa muere, los amigos desaparecen, o se abandona el medio social en que uno ha nacido), el mundo comienza a tambalearse, a perder su objetiva plausibilidad (Berger y Luckmann, 2006, p. 34).

Por lo tanto, es la descomposición de las instituciones sociales la que hace que triunfe el individuo, puesto que éste ya no está normado por las pautas establecidas por los grupos de pertenencia tradicionales, sino por los valores societarios, los cuales le permiten esparcirse por el mundo sin aparente orden, porque como muy bien lo alude Touraine (2005) "el sujeto se forma en la voluntad de escapar a las fuerzas, reglas y poderes que nos impiden ser nosotros mismos, que tratan de reducirnos al estado de un sistema y de su control sobre la actividad, las intenciones y las interacciones de todos. Esas luchas contra lo que nos arrebata el sentido de nuestra existencia son siempre luchas desiguales contra un poder, contra un orden. No hay sujeto [moderno] si no es rebelde, divido entre la cólera y la esperanza" (p. 129).

Miremos cómo [los individuos] administran su experiencia de lógicas de acción relativamente autónomas, en una vida común cada vez menos institucionalizada, cómo tratan de dar nuevo sentido a un mundo social vacío, incierto y efímero; cómo administran su angustia ante riesgos y elecciones inevitables, y su depresión ante los fracasos relativos de su búsqueda de reconocimiento. Y sobre todo, observemos cómo, sobre esta balsa a la deriva, cada quien se esfuerza por ser sí mismo y logra construirse con mayor o menor éxito una identidad personal, llegando a ser y manteniéndose como actor y sujeto de su existencia (Bajoit, 2008, p. 20-21).

Desde esta perspectiva sociológica, se concluye que el Gran ISA se configura entre la opresión y la posibilidad. Esta imagen del individuo, que ya no está definido por las instituciones de pertenecía, de pronto surge y se construye de diversas formas.

\section{El sujeto contemporáneo}

En primer lugar, cabe precisar que la modernidad inicia (con la revolución industrial y la revolución francesa) a finales del siglo XVIII y principios del XIX, la cual continua vigente hasta el día de hoy. Sin embargo, esta modernidad de la que estamos hablando "se define por el hecho de que da fundamentos no sociales a los hechos sociales, impone la sumisión de la sociedad a principios o valores que, en sí mismos, no son sociales" (Touraine, 2005, p. 96).

Este modelo de modernidad nos conduce a la explicación de dos componentes que son necesarios para la existencia de la modernidad que estamos presenciando. El primer principio es la "creencia en la razón y en la acción racional. La ciencia y la tecnología, el cálculo y la precisión, la aplicación de los resultados de la ciencia a dominios cada vez más diversos de nuestra vida y de la sociedad son para nosotros componentes necesarios, y casi evidentes, de la civilización moderna. Lo importante es subrayar que la razón no está basada en la defensa de los intereses colectivos o individuales, sino en sí misma y en un 
concepto de verdad que no se aprehende en términos económicos o políticos. La razón es un fundamento no social de la vida social, mientras que lo religioso o la costumbre se definían en términos sociales, aun cuando se refiriesen a realidades trascendentes puesto que lo sagrado es una realidad social" (Touraine, 2005, p. 96-97).

El segundo principio fundador de la modernidad es el "reconocimiento de los derechos del individuo, es decir, la afirmación de un universalismo que da a todos los individuos los mismos derechos, cualesquiera que sean sus atributos económicos, sociales o políticos. Tal fórmula no apunta a cerrar el debate sobre el lugar de las comunidades en las sociedades individualistas contemporáneas. No impide que lo que llamamos desde 1789 los "derechos del hombre", que están inscritos en diversas constituciones -está en la Declaración Universal de los Derechos Humanos de 1948-, sean reconocidos por todos como una fuerza de superación de todas las comunidades y de todos los principios de orden" (Touraine, 2005, p. 97).

No nos dejemos sorprender por esta aseveración, pues la plena modernidad no puede ser lo opuesto al modelo comunitario, es decir, las formas de organización societarias no pueden ser consideradas únicamente modernas, ya que también las formas comunitarias pensadas como tradicionalistas lo son. En ese sentido, la modernidad no es sólo una forma de vida social, sino un par de fuerzas opuestas y complementarias que dan a una sociedad un completo control de sí misma. Estos dos principios definen bien la modernidad, puesto que rechazan todo orden social que no sea creado por sus propias fuerzas y que esté subordinado a ellas.

El factor que incidió desde el principio en el discurso de la modernidad fue que la sociología clásica se basó en gran medida en un relato sobre esta, sobre una concepción de la evolución entendida como necesidad natural, y en menor grado en una noción de cambio percibido como el producto aleatorio de la historia. La oposición entre la comunidad "tradicionalista" y la sociedad "moderna" fue hecha en la base del pensamiento sociológico clásico, porque el concepto de modernidad era entendido como sinónimo de progreso, cuando en sí las dos nociones no son del mismo orden: la primera noción remite al relato y la segunda a los valores. Este relato llevó durante mucho tiempo al hecho de ver el pasado y la tradición únicamente bajo la perspectiva de una resistencia al cambio, a percibir los desórdenes con el ángulo de la crisis. Esto lo podemos ejemplificar con la representación moderna de la historia del arte, ya que ahí se caracterizan los lazos entre mencionado discurso moderno y el progreso. No requerimos creer en el progreso del arte, Frida Kahlo no es superior a Remedios Varo, ni Enrico Caruso a Luciano Pavarotti, para concebir a la historia de la pintura y de la música como una serie de rupturas, como una serie de innovaciones arrancadas de las tradiciones modernas. Desde este enfoque, el regreso a la tradición es también moderno, siendo que procede de una voluntad libre y racional.

Muchos analistas están tentados de declarar en ruina la modernidad misma y anunciar nuestra entrada en lo posmoderno. Hablar así significa especialmente que se afirma la desaparición de todo principio histórico central de definición del conjunto social. Postura intelectual que tiene consecuencias tan generales y radicales que confiere a los que la adoptan la conciencia de las posibilidades casi infinitas de conceptualización que se conceden y, al mismo tiempo, los graves peligros de desorganización teórica y práctica a los que se exponen. Siempre me he mantenido alejado de esta actitud intelectual, por importante y fecunda que sea (Touraine, 2005, p. 95). 
En pocas palabras, cuando me refiero a la modernidad o a lo moderno, estoy hablando de un continuo proceso de transformación del sujeto contemporáneo, el cual se encuentra en una profunda mutación sociocultural identitaria en la época reciente (de la década de 1970 a la fecha). Por otra parte, entiendo que la modernidad no es sinónimo de progreso (visión evolucionista de la sociedad), ya que dicha percepción es una limitante en las investigaciones sociales contemporáneas.

En segundo lugar, una singularidad de la modernidad es el pluralismo cultural, porque este se define como un estado en donde coexisten en una misma sociedad, personas que viven de diferentes maneras. No estaríamos discutiendo si este no fuera un fenómeno específicamente contemporáneo. Uno podría detectar una u otra variante del pluralismo en casi todas las sociedades, excepto en las arcaicas. El México precolombino se caracterizó por un pluralismo de comunidades originarias que en la actualidad están diseminadas a lo largo y ancho de todo el territorio nacional, mientras que la Europa medieval se distinguió por un pluralismo de estamentos. No obstante, en estos ejemplos las distintas formas de vida estaban vinculadas a un sistema de valores común y la interacción de las comunidades de vida seguía siendo limitada y se mantenía estrictamente regulada. Incluso si definiéramos el pluralismo como un estado en el que es posible encontrar diversas formas de vida dentro de una sociedad, sin que estas se remitan a un sistema de valores común, seríamos capaces de encontrar ejemplos, como el Imperio romano, que en términos económicos y políticos fue una misma sociedad. Sin embargo, incluso en este caso, la interacción de los distintos grupos y personas estaba regulada de tal manera que los esquemas de acción institucionalizados se imponían, aunque estuvieran separados regionalmente. Así pues, los diversos grupos podían interactuar dentro de las esferas institucionales vinculados a su propio sistema de valores y principios.

Si esas regulaciones dejan de aplicarse o ya no pueden ponerse en práctica, entonces se crea una nueva situación que incide de manera importante en el estatus de los sistemas de valores y de las concepciones que se dan por supuestos. Los grupos y comunidades de vida de carácter étnico, religioso y de otro tipo, están divididos de acuerdo con sus identidades, porque no se encuentran separados espacialmente, ni tampoco interactúan sólo en un plano neutral mediante secuencias de acción estrictamente separadas en esferas institucionalizadas.

Esta forma contemporánea de pluralismo constituye, no obstante, la condición básica para la proliferación de crisis identitarias en los sujetos. La pregunta acerca de si el pluralismo actual conduce necesariamente a esta crisis admite varias respuestas. Sin embargo, se podría afirmar con certeza que en países altamente desarrollados (esto es, aquellos donde la modernización del sujeto ha llegado más lejos y donde la forma moderna de pluralismo se ha desarrollado plenamente) los sistemas de valores y las formas identitarias institucionalizadas han dejado de ser patrimonio común de todos los miembros de la sociedad.

El modelo europeo de modernización se ha creado alrededor de una definición de todas las categorías de la organización y el pensamiento social en términos propiamente sociales, es decir, de funciones realizadas por los actores y por las instituciones para asegurar la integración de la sociedad y su capacidad de adaptarse a los cambios necesarios. Este modelo clásico desde hace más de un siglo concede una gran importancia a la representación: se supone que las fuerzas políticas representan a los actores sociales, en particular a las clases sociales. En un dominio diferente, la representación de un personaje consiste en indicar la función social de ese personaje: 
vestidos, posturas, todo debe definir la función social del personaje, y sus características personales se perciben tanto mejor cuando los marcos sociales de quien es representado están indicados de forma más clara. Ahora bien, hoy esos retratos socialmente definidos han muerto (...) el individualismo se impone y se separa de todo entorno social hasta el momento en que toda forma de representación tiende a desaparecer (...) la representación ya no se busca en ninguna parte: se convierte en un signo de mediocridad (Touraine, 2005, p. 72).

Una particularidad del individuo contemporáneo es que este crece en un mundo donde hay múltiples formas de construcción de la identidad, por el libre albedrio que caracteriza a los sujetos. Asimismo, hay una diversidad de realidades en la medida de los universos culturales de cada persona. Aunque el individuo se desarrolla en una comunidad de vida que lo incorpora en un sistema identitario, no cabe suponer que este sea el sistema de sus coetáneos. Esas otras personas pueden haber sido moldeadas por sistemas identitarios completamente distintos dentro de las comunidades de vida en donde crecieron.

Se puede deducir que dichas condiciones promueven la expansión de crisis identitarias en los sujetos modernos. El pensamiento que me retrae tal situación es que cada vez de forma más rápida el modelo de la sociedad se descompone bajo nuestros ojos, mientras que los principios (la creencia en la razón y el reconocimiento de los derechos humanos) de la modernidad tratan de imponerse más directamente.

La declinación del modelo social no se asemeja a la desaparición repentina de una especie viviente, se parece más bien al derrumbe de un monumento cuyos pilares y paredes se agrietan y se erosionan piedra por piedra (Dubet y Martuccelli, 2000, p. 39).

Lejos de percibir este fenómeno como una catástrofe, veo la presencia cada vez más nítida de una modernidad cuyos principios se afirman sobre los escombros de los sistemas sociales. Hoy como en ningún otro momento, los sujetos se encuentran cada vez más ante la responsabilidad de asumirse como seres libres, independientes, autónomos.

Eficaz y brutal, el modo occidental de modernización ha conquistado el mundo en nombre de la sociedad. Pero ha comenzado a perder su fuerza cuando los dominados se han rebelado contra sus amos. El movimiento obrero, primero, los movimientos de liberación nacional después, el movimiento feminista y el movimiento ecologista - fuertemente ligados entre sí - han hecho mella en la dominación total ejercida por la élite dirigente, compuesta de propietarios europeos, adultos y masculinos (...) trabajadores, colonizados, mujeres, minorías de diversos tipos, se han creado entonces una subjetividad (Touraine, 2005, p. 109).

La noción del individuo social tradicional está determinada por su posición en la sociedad, mientras que el individuo moderno escapa a los determinismos sociales en la medida en que es un sujeto autocreador. En otras palabras, un sujeto contemporáneo es más fuerte y consiente de sí mismo, cuando se defiende de aquellos que amenazan su autonomía y su capacidad de asimilarse y ser asimilado como tal. Por ejemplo, en nuestro país el movimiento lésbico/gay lucha porque sean reconocidos todos sus integrantes como sujetos, pero sólo en la Ciudad de México se han ganado ciertos derechos. Esto más que un desanimo en los individuos es un aliciente, porque se han dado cuenta que bien pueden ganar la partida de ajedrez.

En la sociedad moderna, el individuo desarrolla una autonomía sentimental creciente, afirma la legitimidad de sus pasiones y de sus intereses "egoístas", debe efectuar la experiencia de su libertad y de su valor ya que el programa de su vida no está totalmente escrito (Dubet y Martuccelli, 2000, p. 39). 
Por lo tanto, el sujeto moderno presiona con mayor fuerza a la sociedad a través de su singularidad individual, pero el desgaste de esa interacción que se da de manera natural degrada con intensidad la vida social y las pertenencias identitarias. Esta forma de lucha implica, por un lado, no sólo la afirmación de uno mismo como actor social, sino como sujeto personal. Por el otro lado, implica la destrucción de la idea de sociedad. ${ }^{20}$ Aunque parece una catástrofe este evento, conduce a la construcción de la identidad del sujeto contemporáneo.

\section{Construcción de la identidad en el sujeto contemporáneo}

Partimos del precepto de que toda sociedad humana es una construcción del mundo (entendiéndola en términos dialecticos). La sociedad es un fenómeno dialéctico en cuanto que es un producto que se revierte sobre su propio autor. La sociedad es un producto del ser humano. Y no tiene ningún otro ser que el que le confiere la actividad y la conciencia humana. No puede existir el creer colectivo fuera de él mismo. El individuo es producto de la sociedad y viceversa. La sociedad está ahí, antes de que cada individuo nazca y continuará ahí después de su muerte. Sin embargo, dentro de la sociedad, y como resultado de procesos sociales, el individuo se transforma en sujeto a través de las percepciones sociales (el ser humano vive en un mundo de creencias, aunque ese mundo sea asimilado por los demás de forma diferente, él sabe que este mundo posee tales o cuales características comunes a los demás) (Berger y Luckmann, 2006).

El proceso dialéctico se gesta cuando el individuo es productor de conocimiento, y el mundo social su producto. De acuerdo con Berger y Luckmann, este proceso se da en tres momentos: externalización, objetivación e internalización. Sólo podemos alcanzar una visión empíricamente adecuada de la sociedad si comprendemos estas tres etapas como un todo. La externalización es el proceso por el cual las instituciones aparecen fuera del individuo, "persistentes en su realidad "están ahí", existen como realidad externa, el individuo no puede comprenderla por introspección: debe "salir" a conocerlas, así como debe aprender a conocer la naturaleza. El mundo se experimenta como algo distinto a un producto humano". La objetivación es cuando los productos externalizados de la actividad humana alcanzan el carácter de objetividad. "Los significados se materializan permitiendo que el sujeto se vuelva accesible a los conocimientos de su entorno a las experiencias de su práctica cotidiana". Y la internalización es el proceso "por el que el mundo social objetivado vuelve a proyectarse en la conciencia durante la socialización, aprehensión o interpretación inmediata de un acontecimiento objetivo en cuanto expresa significado, o sea, en cuanto es una manifestación de los procesos subjetivos de otros que en consecuencia se vuelven subjetivamente significados para mí" (2006, p. 81).

\footnotetext{
20 "La idea de sociedad no era solamente la construcción de un objeto de estudio en particular, era también una representación colectiva y una filosofía social esforzándose para describir el orden social en un momento en el cual el mundo estaba asistiendo el auge de la revolución industrial, de la revolución democrática, y de la formación de los estados-naciones modernos. La idea de sociedad fue la respuesta brindad por los sociólogos frente a todas estas mutaciones, fue también la manera de reformular las viejas cuestiones concernientes a la acción, el orden y el cambio. La sociología "inventó" la idea de sociedad "frente" a las teologías del siglo clásico, "frente" a las respuestas imbuidas por la idea del contrato del Siglo de las Luces, y "frente" a las respuestas comunitarias de los pensadores contrarrevolucionarios" (Nisbet, citado en Dubet y Martuccelli, 2000, p. 12).
} 
Los tres momentos caracterizan simultáneamente a la sociedad y a cada sector de ella. Estar en sociedad es participar de su dialéctica; el individuo no nace miembro de una sociedad nace con predisposición hacia la sociedad y luego llega a ser miembro de esta; el individuo es inducido a participar en esta dialéctica. El punto de partida de este proceso lo constituye la internalización que compone la base, primero para la comprensión de los propios semejantes y segundo, para la aprehensión del mundo en cuanto al creer significativo y social. Esta aprehensión no resulta de las creaciones autónomas de significado por individuos aislados, sino que comienza cuando el individuo asume el mundo en el que ya viven otros. No sólo vivimos en el mismo mundo, sino que participamos cada uno en el ser del otro, es decir, la actividad del ser humano constructora del mundo es siempre inevitablemente una empresa colectiva (Berger y Luckmann, 2006).

Vamos a ejemplificar este razonamiento. Todo infante se incorpora desde su nacimiento -con sus padres y con otras personas importantes con las que interactúa de manera cotidiana- al ámbito de las relaciones sociales. Estas relaciones se transforman progresivamente en actos regulares, directos y recíprocos. En cierto sentido, el niño es incapaz de realizar una acción en la acepción amplia de la palabra. Aun así, como organismo individuado dispone de las capacidades corporales y de conciencia inherentes a la especie humana, las que emplea en su comportamiento frente a los demás. La acción de sus padres o de sus abuelos respecto del infante está determinada en gran medida por esquemas establecidos institucionalmente. El niño aprende, de manera progresiva, a comprender y a entender las acciones de sus contrapartes. De este modo, es capaz de percibir los actos de los demás como actos típicos a la luz de patrones históricos. Durante este proceso (que Berger y Luckmann designan como socialización primaria), el infante desarrolla progresivamente una identidad personal efímera. Pero, una vez que llega a comprender el sentido de sus acciones y él se considera en principio responsable de ellas, entonces se convierte en sujeto (lo que Berger y Luckmann Ilaman como socialización secundaria). Según dichos autores, eso es lo que constituye la esencia de la identidad del sujeto contemporáneo: el control subjetivo de la acción de la que uno es objetivamente responsable.

Para llegar a este punto el individuo moderno ha pasado diferentes crisis. El sociólogo francés Alain Ehrenberg (1998) ha mencionado que la depresión constituye una de las primeras manifestaciones que se viven a través de sufrimientos específicos engendrados a partir de la mutación contemporánea del individuo.

El imperativo de ser uno mismo, de realizarse, de superarse en esta transformación cultural implica someterse a un cierto grado de crisis identitaria. El individuo que se conforma aplicando las normas de su medio, su cultura y su clase social, ha sido sustituido por la conquista de su identidad personal. Frente a tal reto, muchos de nuestros contemporáneos, en un momento u otro de su vida, o hasta de manera más o menos crónica, sufren un vacío emocional.

De ahí que los rituales necesarios para el reconocimiento identitario "para sí amenazan con no ser más que "mecanismos de defensa" que alimentan manifestaciones neuróticas en las que las crisis de identidad adoptan la forma de sufrimiento psíquico y desafiliación social (Dubar, 2002, p. 176). 
No tenemos duda que la crisis de las formas de representación consideradas como tradicionales han sucumbido por el sistema ideológico heredado en la mutación del individuo moderno. Ya nadie tiene una idea clara e indiscutible sobre la evolución y el progreso social. Ninguno de nosotros cree en una solución milagrosa para los problemas que nos aquejan en la actualidad (Giménez, 1996; Gutiérrez, 2020).

Sin embargo, el tránsito de los jóvenes a este nuevo modelo cultural resultó particularmente difícil, debido a la persistencia tenaz de sus predecesores a mantenerlos atados a través de principios comunes. Pero en el momento que se cuestionaron esas creencias y esos valores, aparecieron los sujetos contemporáneos (Bauman, 2001). ${ }^{21}$

La descomposición de los marcos sociales hace que triunfe el individuo desocializado, pero capaz de combatir tanto el orden social dominante como las fuerzas de la muerte. El individualismo ha estallado pronto en múltiples realidades (Touraine, 2005, p. 129).

Para que se forme esta conciencia del sujeto, nos dice el eminente sociólogo francés Alain Touraine que es necesario que aparezcan y se combinen tres componentes. "En primer lugar, una relación del ser individual consigo mismo, como portador de derechos fundamentales, lo que marca una ruptura con la referencia a principios universalistas, o incluso a una ley divina. El sujeto es su propio fin. En segundo lugar, el sujeto no se forma, hoy como ayer, más que si entra conscientemente en conflicto con las fuerzas dominantes que le niegan el derecho y la posibilidad de actuar como sujeto. Por último, cada uno, en tanto que sujeto, propone una cierta concepción general del individuo" (2005, p. 140-141).

En ese sentido, la construcción identitaria de un individuo consiente y autónomo, un sujeto político y un ciudadano activo no es cosa fácil en nuestros días. Desde el momento en que un individuo moderno no se define como miembro de una institución, ya sea étnica, comunal, familiar, religiosa, etcétera, tiene muchas probabilidades de entrar en crisis. Pero para que no entre en esa coyuntura inestable, es necesario que deba definirse (o redefinirse) de otra forma y encontrar, en él mismo, un principio de representación de sí mismo que pueda compartir con otros. No puede hacerlo sin adherirse a convicciones que pueda argumentar para sí mismo, pero también para los otros.

Como bien lo señala Giménez (1996) la identidad se conforma en la dimensión subjetiva de los individuos sociales modernos, respecto al punto donde se ubiquen entre lo establecido socialmente (el determinismo) y la autonomía (la libertad). En la medida en que simboliza la perspectiva subjetiva de los sujetos sociales sobre sí mismos, el concepto de identidad requiere estar disociado de otras nociones más o menos semejantes como "personalidad" o "carácter social" que conjeturan, por el contrario, el aspecto objetivo del espectador externo o del científico social sobre un actor social determinado. ${ }^{22}$

21 "Jeremy Seabrook recuerda a sus lectores que "el secreto de la sociedad actual reside en el desarrollo de una sensación subjetiva de insuficiencia artificialmente creada, ya que nada podría ser más amenazador para sus principios fundacionales que el hecho de que la gente se declarase satisfecha con lo que posee" (...) Esto ya no es así. Lo único que importa es lo que uno puede hacer, no lo que se debe hacer ni lo que se ha hecho" (Bauman, 2001, p. 124-125). 22 La propuesta inicial de Giménez (1997) "es situar la problemática de la identidad en la intersección de una teoría de la cultura y de una teoría de los actores sociales. O más precisamente, concebir la identidad como elemento de una teoría de la cultura distintivamente internalizada como "habitus" (Bourdieu, 1979, p. 3-6) o como "representaciones sociales" (Abric, 1994, p. 16) por los actores sociales, sean estos individuales o colectivos. De este modo, la identidad no sería más que el lado subjetivo de la cultura considerada bajo el ángulo de su función distintiva" (p. 10-11). 
Se puede decir entonces que la identidad en el sujeto contemporáneo no se reduce a un conjunto de datos objetivos, sino a una selección de operaciones subjetivas. Básicamente es reconocerse en algo que posiblemente sólo coincide en parte con lo que uno realmente es. El resultado de transformar un dato en valor constituye la identidad. No es lo que uno verdaderamente es, sino la percepción que cada uno tiene sobre sí mismo.

\section{REFERENCIAS}

Bajoit, G. (2009). La tiranía del gran ISA. Cultura y representaciones sociales, 3(6), 9-24. Recuperado de http://www.culturayrs.unam.mx/index.php/CRS/article/view/542/549

Bajoit, G. (2008). La renovación de la sociología contemporánea. Cultura y representaciones sociales, 3(5), 9-31. Recuperado de

http://www.culturayrs.unam.mx/index.php/CRS/article/view/534/541

Bajoit, G. (2003). Todo cambia: análisis sociológico del cambio social y cultural en las sociedades contemporáneas. Chile: Ediciones LOM.

Bartolomé, M. A. (2004). Gente de costumbre y gente de razón. Las identidades étnicas en México. México: Siglo XXI.

Bauman, Z. (2007). Vida de consumo. México: Fondo de Cultura Económica.

Bauman, Z. (2004). Modernidad líquida. Argentina: Fondo de Cultura Económica.

Bauman, Z. (2001). La globalización. México: Fondo de Cultura Económica.

Bauman, Z. (1996). Teoría sociológica de la posmodernidad. Espiral, 2(5), 81-102. Recuperado de https:// www.redalyc.org/pdf/138/13820504.pdf

Bell, D. (1991). El advenimiento de la sociedad postindustrial. España: Alianza.

Berger, P. y Luckmann, T. (2006). La construcción social de la realidad. Argentina: Amorrortu.

Berger, P. y Luckmann, T. (1997). Modernidad, pluralismo y crisis de sentido. La orientación del hombre moderno. España: Paidós.

Bourdieu, P. (2007). El sentido práctico. Argentina: Siglo XXI.

Castells, M. (2005). La era de la información, economía, sociedad y cultura, Vol. I: La sociedad red. México: Siglo XXI.

Castells, M. (2003). La era de la información, economía, sociedad y cultura, Vol. II: El poder de la identidad. México: Siglo XXI.

Castells, M. (2001). La era de la información, economía, sociedad y cultura, Vol. Ill: Fin de milenio. México: Siglo XXI.

Derrida, J. (1986). De la gramatología. México: Siglo XXI.

Dubar, C. (2002). La crisis de las identidades: la interacción de una mutación. España: Bellaterra.

Dubet, F. (2006). El declive de la institución. España: Gedisa.

Dubet, F. y Martuccelli, D. (2000). En qué sociedad vivimos. Argentina: Losada.

Douglas, M. (1996). Cómo piensan las instituciones. España: Alianza.

Durkheim, É. (2001). La educación moral. México: Colofón.

Durkheim, É. (1998). Las formas elementales de la vida religiosa. México: Colofón.

Durkheim, É. (1997). Las reglas del método sociológico. México: Colofón.

Durkheim, É. (1990). Educación y sociología. España: Ediciones 62.

Durkheim, É. (1976). Educación como socialización. España: Ediciones Sígueme. 
Ehrenberg, A. (1998) La fatiga de ser uno mismo. Depresión y sociedad. Argentina: Nueva Visión. Fichter, J. (1994). Sociología. España: Herder.

García, N. (1990). Culturas Híbridas; estrategias para entrar y salir de la modernidad. México: Grijalbo. Giménez,G.(2002).IntroducciónalasociologíadePierreBourdieu.Colección PedagógicaUniversitaria,37(38), 1-11. Recuperado de https://biblat.unam.mx/hevila/Coleccionpedagogicauniversitaria/2002/ no37-38/1.pdf

Giménez, G. (1997). Materiales para una teoría de las identidades sociales. Frontera Norte, 9(18), 9-28. Recuperado de https://fronteranorte.colef.mx/index.php/fronteranorte/article/viewFile/1441/891

Giménez, G. (1996). La identidad social o el retorno del sujeto en sociología. En Identidad: análisis y teoría, simbolismo, sociedades complejas, nacionalismo y etnicidad. México: UNAM.

Gutiérrez, Á. A. (2020). Juventud, crisis identitaria y globalización de la cultura. Perfiles de las Ciencias Sociales, 8(15), 345-364. Recuperado de https://revistas.ujat.mx/index.php/perfiles/article/ view/3919/2956

Gutiérrez, Á. A. (2019). Panorama étnico religioso en México. Ecos Sociales, 7(19), 672-683. Recuperado de http://www.revistas.ujat.mx/index.php/ecosoc/article/view/3211/2407

Gutiérrez, Á. A. (2017). Paradoja de la reivindicación en la identidad étnica zoque. Ecos Sociales, 5(15), 437-448. Recuperado de http://www.revistas.ujat.mx/index.php/ecosoc/article/view/2092/1683

Habermas, J. (1992a). Teoría de la acción comunicativa I. Racionalidad de la acción y racionalización social. España: Taurus.

Habermas, J. (1992b). Teoría de la acción comunicativa II. Crítica de la razón funcionalista. España: Taurus. Harvey, D. (1998). La condición de la posmodernidad. Investigación sobre los orígenes del cambio cultural. Argentina: Amorrortu.

Inglehart, R. (2005). Modernización y cambio cultural: la persistencia de los valores tradicionales. Cuadernos del Mediterráneo, 5, 21-32. Recuperado de https://dialnet.unirioja.es/servlet/ articulo?codigo $=1395031$

Kaminsky, G. (2001). Socialización. México: Trillas.

Lyotard, J. F. (2006). La condición postmoderna. España: Cátedra.

Martín-Barbero, J. (2004). Crisis identitarias y transformaciones de la subjetividad. En Debates sobre el sujeto. Perspectivas contemporáneas. Colombia: Siglo del Hombre Editores.

Mercado, A. y Hernández, A. (2010). El proceso de construcción de la identidad colectiva. Convergencia. Revista de Ciencias Sociales, 53, 229-251. https://www.redalyc.org/pdf/105/10513135010.pdf

Radcliffe-Brown, A. (1980). Estructura y función en la sociedad primitiva. España: Ediciones 62.

Real Academia Española (2011). Diccionario de la lengua española, vigésima primera edición (formato EPUB), Real Academia Española, Madrid, España.

Salles, V. y Valenzuela, J. M. (1997). En muchos lugares y todos los días. Vírgenes, santos y niños dios. Mística y religiosidad popular en Xochimilco. México: El Colegio de México.

Sauvé, L. (1999). La educación ambiental entre la modernidad y la posmodernidad: en busca de un marco de referencia educativo integrador. Tópicos en Educación Ambiental, 1(2), 7-25. Recuperado de http://www.anea.org.mx/Topicos/T\%202/Pagina\%2007-25.PDF

Tejeda, J. L. (2005). La búsqueda de la identidad. Revista Interamericana de Educación de Adultos, 27(2), 172-186. Recuperado de https://www.redalyc.org/pdf/4575/457545128008.pdf

Touraine, A. (2005). Un nuevo paradigma para comprender el mundo hoy. España: Paidós.

Touraine, A. (2004). Critica de la modernidad. México: Fondo de Cultura Económica.

Weber, M. (1987). Economía y sociedad. México: Fondo de Cultura Económica. 